\title{
Cemiplimab in locally advanced basal cell carcinoma after hedgehog inhibitor therapy: an open-label, multi-centre, single-arm, phase 2 trial
}

\author{
Alexander J Stratigos, Aleksandar Sekulic, Ketty Peris, Oliver Bechter, Sorilla Prey, Martin Kaatz, Karl D Lewis, Nicole Basset-Seguin, \\ Anne Lynn S Chang, Stéphane Dalle, Almudena Fernandez Orland, Lisa Licitra, Caroline Robert, Claas Ulrich, Axel Hauschild, Michael R Migden, \\ Reinhard Dummer, Siyu Li, Suk-Young Yoo, Kosalai Mohan, Ebony Coates, Vladimir Jankovic, Nathalie Fiaschi, Emmanuel Okoye, \\ loannis D Bassukas, Carmen Loquai, Vincenzo De Giorgi, Zeynep Eroglu, Ralf Gutzmer, Jens Ulrich, Susana Puig, Frank Seebach, Gavin Thurston, \\ David M Weinreich, George D Yancopoulos, Israel Lowy, Timothy Bowler, Matthew G Fury
}

\section{Summary}

Background Before February, 2021, there was no standard treatment regimen for locally advanced basal cell carcinoma after first-line hedgehog inhibitor (HHI) therapy. Cemiplimab, a PD-1 antibody, is approved for treatment of advanced cutaneous squamous cell carcinoma and has shown clinical activity as monotherapy in first-line non-small-cell lung cancer. Here, we present the primary analysis data of cemiplimab in patients with locally advanced basal cell carcinoma after HHI therapy.

Methods We did an open-label, multicentre, single-arm, phase 2 trial across 38 outpatient clinics, primarily at academic medical centres, in Canada, Europe, and the USA. Eligible patients (aged $\geq 18$ years and with an Eastern Cooperative Oncology Group performance status of 0 or 1) with a histologically confirmed diagnosis of metastatic basal cell carcinoma (group 1) or locally advanced basal cell carcinoma (group 2) who had progressed on or were intolerant to previous HHI therapy were enrolled. Patients were not candidates for further HHI therapy due to progression of disease on or intolerance to previous HHI therapy or having no better than stable disease after 9 months on HHI therapy. Patients received cemiplimab $350 \mathrm{mg}$ intravenously every 3 weeks for up to 93 weeks or until progression or unacceptable toxicity. The primary endpoint was objective response by independent centra review. Analyses were done as per the intention-to-treat principle. The safety analysis comprised all patients who received at least one dose of cemiplimab. The primary analysis is reported only for group 2; group 1 data have not reached maturity and will be reported when the timepoint, according to the statistical analysis plan, has been reached. This study is registered with ClinicalTrials.gov, NCT03132636, and is no longer recruiting new participants.

Findings Between Nov 16, 2017, and Jan 7, 2019, 84 patients were enrolled and treated with cemiplimab. At data cutoff on Feb 17, 2020, median duration of follow-up was 15 months (IQR 8-18). An objective response per independent central review was observed in 26 (31\%; 95\% CI 21-42) of 84 patients, including two partial responses that emerged at tumour assessments before the data cutoff and were confirmed by tumour assessments done subsequent to the data cutoff. The best overall response was five (6\%) patients with a complete response and $21(25 \%)$ with a partial response. Grade 3-4 treatment-emergent adverse events occurred in 40 (48\%) of 84 patients; the most common were hypertension (four [5\%] of 84 patients) and colitis (four [5\%]). Serious treatment-emergent adverse events occurred in $29(35 \%)$ of 84 patients. There were no treatment-related deaths.

Interpretation Cemiplimab exhibited clinically meaningful antitumour activity and an acceptable safety profile in patients with locally advanced basal cell carcinoma after HHI therapy.

Funding Regeneron Pharmaceuticals and Sanofi.

Copyright (C) 2021 Elsevier Ltd. All rights reserved.

\section{Introduction}

Basal cell carcinoma is the most common human malignancy worldwide. ${ }^{1}$ Exposure to ultraviolet light is a major risk factor. ${ }^{2}$ Surgery and other local modality treatments such as radiotherapy or topical drugs are curative options for most patients, but a small percentage develop advanced basal cell carcinoma for which systemic therapy is indicated. ${ }^{3}$ Vismodegib is a hedgehog signalling pathway inhibitor (HHI) that is approved for treatment of patients with metastatic basal cell carcinoma or locally advanced basal cell carcinoma who are not candidates for curative surgery or curative radiotherapy. Sonidegib is another $\mathrm{HHI}$ that is approved for the treatment of locally advanced basal cell carcinoma only. In phase 2 studies, vismodegib and sonidegib showed objective response rates of $30-60 \%$ in advanced basal cell carcinoma. ${ }^{4-7}$ However, most patients progress on or are intolerant to HHI therapy and until
Lancet Oncol 2021

Published Online May 14, 2021 https://doi.org/10.1016/ S1470-2045(21)00126-1

First Department of Dermatology-Venereology, Andreas Syggros HospitalNational and Kapodistrian University of Athens, Athens, Greece (Prof A J Stratigos MD); Arizona Mayo Clinic, Phoenix AZ, USA (Prof A Sekulic MD); Catholic University of the Sacred Heart and Fondazione Policlinico Universitario A Gemelli-IRCCS, Rome, Italy (Prof K Peris MD); Department of General Medical Oncology, University Hospitals, Leuven, Belgium (Prof O Bechter MD); Department of Dermatology, University Medical Center,

Bordeaux, France (S Prey MD); SRH Wald-Klinikum Gera, Gera, Germany (M Kaatz MD); University of Colorado Cancer Center, Aurora, CO, USA (Prof K D Lewis MD); Hopital Saint-Louis, Paris, France (Prof N Basset-Seguin MD); Department of Dermatology, Stanford University School of Medicine, Stanford, CA, USA (Prof A LS Chang MD); Department of Dermatology, HCL Cancer Institute, Lyon Cancer Research Center, Lyon, France (Prof S Dalle MD): Hospital Universitario Virgen Macarena, Seville, Spain (A F Orland MD); Fondazione IRCCS, Istituto Nazionale dei Tumori, Milan, Italy (L Licitra MD); Department of Oncology and HematologyOncology, University of Milan, Milan, Italy (L Licitra); Dermatology Unit, Gustave Roussy Cancer Center, Villejuif France (C Robert MD); ParisSaclay University, Villejuif, France (C Robert); ChariteUniversitiitsmedizin Berlin, 
Berlin, Germany (CUlrich MD); Department of Dermatology, University of Kiel, Kiel, Germany (Prof A Hauschild MD); The University of Texas MD Anderson Cancer Center, Houston, TX, USA (Prof M R Migden MD); Department of Dermatology, University Hospital Zurich, Zurich, Switzerland (Prof R Dummer MD); Regeneron Pharmaceuticals, Basking Ridge, NJ, USA (S Li PhD, S-Y Yoo PhD); Regeneron Pharmaceuticals, Tarrytown, NY, USA (K Mohan PhD, E Coates MHA, V Jankovic MD, N Fiaschi PhD, E Okoye MBBS, F Seebach MD, G Thurston PhD, D M Weinreich MD,

G D Yancopoulos MD, I Lowy MD, T Bowler MD, M G Fury MD); University of loánnina, loánnina, Greece (Prof I D Bassukas MD); Department of Dermatology, University Medical Center Mainz, Mainz, Germany (C Loquai MD); Department of Dermatology, University of Florence, Florence, Italy (V De Giorgi MD); Department of Cutaneous Oncology, Moffitt Cancer Center, Tampa, FL, USA (Z Eroglu MD); Skin Cancer Center Hannover, Department of Dermatology and Allergy, Hannover Medical

School, Hannover, Germany (Prof R Gutzmer MD); Skin Cancer Center,

Department of Dermatology, Harz Clinics, Quedlinburg, Germany (Prof JUlrich MD); Hospital Clínic de Barcelona, Institut d'Investigacions Biomediques August Pi I Sunyer (IDIBAPS), Universitat de Barcelona, and Centro de Investigación Biomédica en Red de Enfermedadees Raras (CIBERER), Instituto de Salud Carlos III, Barcelona, Spain (S Puig MD)

Correspondence to: Prof Alexander J Stratigos, Andreas Syggros HospitalNational and Kapodistrian University of Athens, Athens 16121, Greece alstrat@med.uoa.gr

See Online for appendix

\section{Research in context}

\section{Evidence before this study}

For most patients with basal cell carcinoma, surgery and other local modality treatments are curative options. Systemic therapy is indicated for a small percentage of patients who develop advanced basal cell carcinoma. The hedgehog signalling pathway inhibitors (HHIs), vismodegib (approved for advanced basal cell carcinoma) and sonidegib (approved for locally advanced basal cell carcinoma only), have shown objective responses in 30-60\% of patients with advanced basal cell carcinoma. However, most patients progress on or are intolerant to $\mathrm{HHI}$ therapy and until Feb 9, 2021, when cemiplimab was approved by the US Food and Drug Administration fully for patients with locally advanced basal cell carcinoma, and accelerated for patients with metastatic basal cell carcinoma, after HHI treatment, or for whom HHIs are not appropriate, there was no standard second-line treatment option for this patient population. Basal cell carcinoma has one of the highest tumour mutational burdens of any malignancy, a characteristic that is associated with response to PD-1 blockade. We searched PubMed with no language restrictions from May 1, 2012, to May 1, 2017, with the search terms "locally advanced basal cell carcinoma" OR "metastatic basal cell carcinoma" OR "advanced basal cell carcinoma". After excluding publications from first-line advanced basal cell carcinoma treatment, we found only one relevant publication: a study of nine patients with advanced basal cell carcinoma who were resistant to vismodegib but had no objective response to subsequent sonidegib. We also found a case report that showed clinical benefit with pembrolizumab after progression on $\mathrm{HHI}$ therapy. Another case report (from the phase 1 trial stage of the present study) published within the timeframe of the search but not included in the search results, showed a durable partial response to cemiplimab in a patient with metastatic basal cell carcinoma.

\section{Added value of this study}

Results from this study showed that cemiplimab is an active treatment option for patients with locally advanced basal cell carcinoma who had progressed on or are intolerant to $\mathrm{HHI}$ therapy. The safety profile of cemiplimab in this study was also acceptable. This is the first study to show activity of a systemic therapy in locally advanced basal cell carcinoma after $\mathrm{HHI}$ therapy, therefore addressing an unmet need in this patient population.

\section{Implications of all the available evidence}

Our findings support the indication in the USA of cemiplimab for patients with locally advanced basal cell carcinoma after $\mathrm{HHI}$ therapy or for whom HHI therapies are not appropriate.
Feb 9, 2021, when cemiplimab was approved by the US Food and Drug Administration fully for patients with locally advanced basal cell carcinoma, and accelerated for patients with metastatic basal cell carcinoma, after HHI treatment, or for whom HHIs are not appropriate, there was no standard second-line treatment option for these patients. ${ }^{4,8}$

Basal cell carcinoma has one of the highest mutational burdens of any human malignancy. ${ }^{9}$ Tumour types with high mutational burden are generally more responsive to PD-1 blockade. ${ }^{10}$ The risk of basal cell carcinoma is ten times higher in recipients of solid organ transplants (and other groups with induced or acquired lack of immunosurveillance) than in the general population, suggesting that adaptive immune responses are specifically important in this disease. ${ }^{11}$

Cemiplimab is a highly potent, fully human, hingestabilised, immunoglobulin G4 monoclonal antibody directed against PD-1, derived using VelocImmune technology, ${ }^{12,13}$ and is approved for the treatment of patients with metastatic cutaneous squamous cell carcinoma (CSCC) or locally advanced CSCC who are not candidates for curative surgery or curative radiation. ${ }^{14-16}$ Cemiplimab has recently been shown to have profound clinical activity as monotherapy in first-line non-small-cell lung cancer with $50 \%$ or more PD-L1 expression. ${ }^{17}$ In the first-inhuman study of cemiplimab, a durable partial response was observed in a patient with metastatic basal cell carcinoma treated with cemiplimab..$^{18}$ In this Article, we present the primary analysis of the phase 2 study of cemiplimab monotherapy in patients with locally advanced basal cell carcinoma after HHI therapy.

\section{Methods}

\section{Study design and participants}

We did an open-label, multicentre, single-arm, phase 2 trial of cemiplimab monotherapy across 38 outpatient clinics, primarily at academic medical centres, in Canada, Europe, and the USA (appendix pp 3-5). The study was designed to include adult patients who had advanced basal cell carcinoma, that is, metastatic basal cell carcinoma (group 1) or locally advanced basal cell carcinoma (group 2). The prespecified timing of the primary analysis was reached for the locally advanced basal cell carcinoma cohort. Thus, we report results for group 2 here. Results of the metastatic basal cell carcinoma (group 1) cohort are not included in this Article because the data are interim, and the time point for the primary analysis (according to the statistical analysis plan) has not yet been reached.

Patients aged 18 years or older with an Eastern Cooperative Oncology Group performance status of 0 or 1 were included if they had histologically confirmed diagnosis of locally advanced basal cell carcinoma; were not candidates for further HHI therapy due to progression of disease on or intolerance to previous HHI therapy or 
having no better than stable disease after 9 months on HHI therapy; and had at least one baseline lesion measurable by digital medical photography per modified WHO criteria or by radiological imaging (CT or MRI) as per the Response Evaluation Criteria in Solid Tumors (RECIST) version 1.1 criteria. $^{19}$ Outcomes of previous HHI therapy (progressive disease, stable disease, or intolerance) was per investigator assessment. Eligible patients underwent multidisciplinary assessments during screening to establish that they were not candidates for curative surgery or curative radiotherapy. Key exclusion criteria were ongoing or recent (within 5 years) evidence of substantial autoimmune disease requiring systemic immunosuppression; previous treatment with an antiPD-1 or an anti-PD-L1 drug; concurrent malignancy other than basal cell carcinoma or history of malignancy other than basal cell carcinoma within 3 years of date of first planned dose of cemiplimab, except for tumours with negligible risk of metastasis or death. Full details about the eligibility criteria can be found in the study protocol (appendix p 20).

The study protocols and all amendments were approved by the institutional review board at each participating study site. The study was done in accordance with the principles of the Declaration of Helsinki and with Good Clinical Practice guidelines as defined by the International Conference on Harmonisation. All patients provided written, informed consent before enrolment.

Relevant protocol amendments included extension of post-treatment follow-up for an additional 1 year, for a total of approximately 1.5 years after completion of the treatment at the end of extended follow-up (amendment date July 29, 2019); an update to the length of treatment period to nine cycles; revision of tumour response assessment timing; revision that all patients should be followed up for a minimum of 6 months from onset of response for the analysis of duration of response; and an update that analysis of duration of response was to be done after all responding patients had been followed up for a minimum of 12 months from onset of response (amendment date March 23, 2017). The full protocol amendment history is provided in the protocol.

\section{Procedures}

After a screening period of up to 28 days, patients received cemiplimab $350 \mathrm{mg}$ intravenously every 3 weeks; therapy consisted of cemiplimab infusions every 3 weeks for a planned 93 weeks. Patients received treatment until the 93-week treatment period was complete or until disease progression, unacceptable toxicity, or withdrawal of consent.

Tumour response assessments were done every 9 weeks for the first five assessments, and every 12 weeks thereafter. Baseline assessments included digital medical photography and radiological imaging (CT or MRI) of all target lesions. For each response assessment, repeat of the same photographic and radiological assessments completed at baseline were encouraged. In cases where the investigator's opinion of baseline imaging (photography and radiology) was that the tumour was comprehensively assessed by one modality (photography or radiology), post-baseline assessments could consist of only photography or radiology. All responses were required to be confirmed by two separate tumour assessments, at least 4 weeks apart. To establish a complete response per independent central review (ICR), biopsy of regressed target lesion documenting histological negativity was required. If the last tumour assessment before the data cutoff was the first documentation of response, the centrally reviewed tumour assessment subsequent to data cutoff was allowed to confirm response status.

For patients with locally advanced basal cell carcinoma, modified WHO criteria and RECIST 1.1 criteria $^{19}$ were used for digital medical photography and radiological imaging, respectively. An independent composite review committee reviewed digital medical photography, radiology, and pathology reports from on-treatment biopsies (if any) to adjudicate response status for each tumour assessment (if the independent composite review committee determined that a patient did not have an evaluable target lesion at baseline, the patient was still included in primary and secondary efficacy assessments because the investigator's opinion was that each patient did have a measurable lesion at baseline and was appropriate to enrol).

Safety assessments included reporting of treatmentemergent adverse events, measurement of vital signs, physical examination, electrocardiograms, and laboratory monitoring. Safety assessment was done continuously from initiation of study treatment until 105 days after the last study treatment. Laboratory tests for blood chemistry and haematology were done on the same day as the administration of each study treatment and at the end of the study (30 days after the last dose of cemiplimab; full details are in the study protocol). Adverse events were coded according to the preferred terms of the Medical Dictionary for Regulatory Activities (version 22.1). The severity of treatment-emergent adverse events was graded according to the National Cancer Institute Common Terminology Criteria for Adverse Events (version 4.03).

Treatment interruptions were recommended for treatment-related adverse events of grade 3 or higher, and for grade 2 uveitis. Patients were considered for resumption of study treatment, at investigator's discretion, once treatment-related adverse events had resolved to grade 1 or baseline. Up to two cemiplimab dose reductions were allowed (dose level 1, cemiplimab $120 \mathrm{mg}$ every 3 weeks; dose level 2, cemiplimab 60 mg every 3 weeks).

The study sponsor used a customised Medical Dictionary for Regulatory Activities query to identify immune-related adverse events. Treatment-related adverse events included in the sponsor list of immune-related adverse event preferred terms were considered potential immunerelated adverse events. To avoid underestimating the 
frequency of certain immune-related adverse events, preferred terms describing the same medical concept were combined into composite terms. Identified immunerelated adverse events were defined as potential immunerelated adverse events requiring treatment with systemic corticosteroids or other immunosuppressants or events that were immune-related endocrinopathies.

Pretreatment biopsies of tumours were used to explore potential biomarkers including expression of selected proteins (PD-L1 and MHC class I by immunohistochemistry, and tumour mutational burden [TMB]). High and low TMB were categorised as 10 or more and less than 10 mutations per megabase (mut/Mb), respectively. MHC-I expression scoring was based on quantitative image analysis, and the MHC-I positive percentage was calculated as the number of MHC-I positive tumour cells divided by the total number of tumour cells, multiplied by 100 . PD-L1 expression was quantified as the percentage of tumour cells with detectable PD-L1 membrane staining (tumour proportion score). Additional information on biomarker methodology can be found in the appendix ( $\mathrm{p} 6)$.

Major deviations occurring in more than one patient were: administration of other anticancer treatment within 30 days of initiating cemiplimab treatment; no measurable tumour lesion at baseline; creatinine phosphokinase concentration not measured before first dose of cemiplimab; tumour sample not received in central laboratory before first dose of cemiplimab; or serious adverse events or adverse events of special interest not reported within $24 \mathrm{~h}$ (appendix $\mathrm{p} 7$ ).

\section{Outcomes}

The primary endpoint was objective response, defined as the proportion of patients with the best overall response of complete or partial response, as assessed by ICR.

Secondary endpoints included objective response according to investigator review; duration of response (by ICR and investigator review), defined as the time between first measurement of complete or partial response and the first date of recurrent or progressive disease or death; progression-free survival (by central and investigator review), defined as the time between start of treatment and the first date of recurrent or progressive disease or death from any cause; overall survival, defined as the time between the start of treatment and death from any cause; proportion of patients attaining best response of complete response by ICR; time to response, defined as the time between start of treatment and the first best response of complete or partial response (whichever comes first); and safety and tolerability of cemiplimab.

Additional secondary outcome measures included disease control, defined as the proportion of patients with a best response of complete response, partial response, stable disease, or non-partial response or non-progressive disease at the first evaluable tumour assessment, scheduled to occur at week 9 (defined as 56 days to account for visit windows in the protocol); and durable disease control, defined as the proportion of patients without progressive disease for at least 182 days. Other secondary outcomes were pharmacokinetics and immunogenicity of cemiplimab, and effect of cemiplimab on patient-reported quality of life; the data from these will be published when the analyses are completed. Protocol-defined exploratory outcomes included associations between clinical activity of cemiplimab and PD-L1 immunohistochemistry, TMB assessments, or MHC-I immunohistochemistry.

\section{Statistical analysis}

Primary and secondary clinical activity outcomes were assessed according to the intention-to-treat principle among enrolled patients. All patients who received at least one dose of cemiplimab were assessed for safety. The timing of the primary analysis was prespecified to provide the opportunity for all responses to have a duration of at least 6 months. The protocol specified that the last patient with locally advanced basal cell carcinoma enrolled would be followed up for 27 weeks (cycles 1-3) for the opportunity to develop a response, plus an additional 30 weeks (cycles 4-6) to observe duration of response. Therefore, the prespecified data cutoff date was approximately 57 weeks after last patient first visit. The data cutoff date was Feb 17, 2020.

For the primary endpoint of objective response, the statistical hypothesis was that patients treated with cemiplimab would have a response rate representing a clinically meaningful treatment, corresponding to values published for previous studies of treatment with HHIs. A sample size of 80 patients with locally advanced basal cell carcinoma was estimated to provide at least $85 \%$ power to reject a null hypothesis of an objective response in $20 \%$ of patients at a two-sided significance level of $5 \%$ if the true objective response is observed in $35 \%$ of patients. To account for patients who prematurely withdraw from the study, the sample size was increased by $5 \%$. Therefore, the total planned sample size was 84 patients. The primary analysis was based on the binomial exact confidence interval (CI) approach, with CI calculated using the Clopper-Pearson method. ${ }^{20}$ The null hypothesis (objective response in $20 \%$ of patients or less) would be excluded using the lower limit of $95 \%$ CI if the observed objective response was in $30 \%$ of patients or more. These thresholds were selected as a guide to assess meaningful benefit, as there were no approved systemic treatments available in the second-line setting at the time of the study.

The secondary endpoints, duration of response, progression-free survival, and overall survival, were estimated using the Kaplan-Meier method. For duration of response in patients with response, and for progressionfree survival, patients without disease progression and who did not die were censored at the time of their last valid tumour assessment (unless new anticancer therapy was started); patients without any evaluable post-baseline 


\begin{tabular}{|lc|}
\hline & Patients (n=84) \\
\hline Median age, years & $70(61-79)$ \\
\hline Age $\geq 65$ years & $53(63 \%)$ \\
Sex & \\
Male & $56(67 \%)$ \\
Female & $28(33 \%)$ \\
\hline Eastern Cooperative Oncology Group performance status score \\
0 & $51(61 \%)$ \\
1 & $33(39 \%)$ \\
Patients with previous cancer-related radiotherapy & $42(50 \%)$ \\
\hline Patients with previous HHI & \\
Vismodegib & $79(94 \%)$ \\
Sonidegib & $14(17 \%)$ \\
Vismodegib plus sonidegib & $9(11 \%)$ \\
Reason for discontinuation of previous HHI* & \\
Progression of disease on HHI & $60(71 \%)$ \\
Intolerant to previous HHI therapy & $32(38 \%)$ \\
$\quad$ Intolerant to vismodegib & $32(38 \%)$ \\
$\quad$ Intolerant to sonidegib & $4(5 \%)$ \\
No better than stable disease after 9 months on & $7(8 \%)$ \\
HHI therapy & \\
\hline Primary basal cell carcinoma site & $75(89 \%)$ \\
Head and neck & $7(8 \%)$ \\
Trunk & $2(2 \%)$ \\
Arm or leg & \\
Data are median (IQR) or $n$ (\%). HHI=hedgehog inhibitor. ${ }^{*}$ The sum is more than \\
84 because some patients had more than one reason for discontinuation. \\
\hline Table 1: Baseline patient characteristics & \\
\hline
\end{tabular}

tumour assessment and who did not die were censored on the date of first study treatment. For overall survival, patients without a survival event were censored at the time of last known survival. The secondary endpoints disease control and durable disease control were summarised with two-sided 95\% exact binomial CIs using the ClopperPearson method; time to response was summarised descriptively. Prespecified subgroup analyses of the primary endpoint, objective response, by baseline characteristics (sex, age, and previous HHI therapy) were also done. Prespecified exploratory biomarker outcomes were summarised descriptively. TMB and MHC-I were also displayed by box and scatter plots according to the clinical activity of cemiplimab. Statistical analyses were done with SAS (version 9.4). This study is registered with ClinicalTrials.gov, NCT03132636.

\section{Role of the funding source}

The study was sponsored by Regeneron Pharmaceuticals and Sanofi and was designed by employees of Regeneron Pharmaceuticals, in collaboration with the investigators. Data from the study were collected by investigators, analysed by statisticians employed by the sponsors, and interpreted by the authors, including employees of the sponsors. The first draft of the manuscript was prepared by a medical writer (funded by the sponsors) and was

\begin{tabular}{|c|c|}
\hline & Patients $(n=84)$ \\
\hline Objective response & $26(31 \% ; 21-42)^{*}$ \\
\hline \multicolumn{2}{|l|}{ Best overall response } \\
\hline Complete response & $5(6 \%)$ \\
\hline Partial response & $21(25 \%)$ \\
\hline Stable disease & $41(49 \%)$ \\
\hline Progressive disease & $9(11 \%)$ \\
\hline Not evaluable $\dagger$ & $8(10 \%)$ \\
\hline Disease control & $67(80 \% ; 70-88)$ \\
\hline Durable disease control & $50(60 \% ; 48-70)$ \\
\hline Median time to response, months $\ddagger$ & $4 \cdot 3(4 \cdot 2-7 \cdot 2)$ \\
\hline \multicolumn{2}{|l|}{ Observed duration of response $\ddagger$} \\
\hline Range, months & $2-21$ \\
\hline$\geq 6$ months & $19(79 \%)$ \\
\hline$\geq 12$ months & $11(46 \%)$ \\
\hline \multicolumn{2}{|c|}{ Kaplan-Meier estimation of duration response $\ddagger$} \\
\hline Median & Not reached \\
\hline Remained in response at 6 months & $91 \%(68-98)$ \\
\hline Remained in response at 12 months & $85 \%(61-95)$ \\
\hline \multicolumn{2}{|c|}{$\begin{array}{l}\text { Data are n }(\% ; 95 \% \mathrm{Cl}), \mathrm{n}(\%) \text {, median (IQR), or range (where specified). * }{ }^{*} \text { bjective } \\
\text { response per independent central review includes two partial responses that } \\
\text { emerged at tumour assessments before the data cutoff and were confirmed by } \\
\text { tumour assessments done subsequent to the data cutoff. †Of the eight patients } \\
\text { who were not evaluable, four did not have any post-baseline tumour } \\
\text { assessments, three patients were not considered to have evaluable lesions by } \\
\text { either photographic or radiological assessment methods per the independent } \\
\text { composite review committee, and one patient had a second target lesion not } \\
\text { imaged after baseline. fData shown are for patients with a confirmed complete } \\
\text { response or partial response; duration of response was calculated for all patients } \\
\text { with a confirmed response prior to the data cutoff. }\end{array}$} \\
\hline $\begin{array}{l}\text { Table 2: Tumour response and duration } \\
\text { central review }\end{array}$ & independent \\
\hline
\end{tabular}

based on authors' comments on the manuscript outline, which was also prepared by the medical writer. Thereafter, the first draft was critically reviewed and revised by the authors, including employees of the sponsors.

\section{Results}

Between Nov 16, 2017, and Jan 7, 2019, 84 patients with locally advanced basal cell carcinoma were enrolled, treated with cemiplimab, and included in the analyses. The primary tumour site was the head and neck and the most common reason for discontinuation of previous HHI therapy was disease progression (table 1).

At the time of data cutoff (Feb 17, 2020), 19 patients remained on treatment, 13 had completed the planned treatment of 93 weeks, and 52 had discontinued, mainly due to disease progression $(n=29$; appendix $p$ 8). The median number of administered doses was 15 (IQR 8-24). Median duration of exposure to cemiplimab was 47 weeks (IQR 27-80). Median duration of follow-up was 15 months (IQR 8-18).

By ICR, an objective response was observed in 26 (31\%, $95 \%$ CI $21-42$ ) of 84 patients, including five (6\%) complete responses and 21 (25\%) partial responses (table 2). The objective response per ICR included two partial responses 


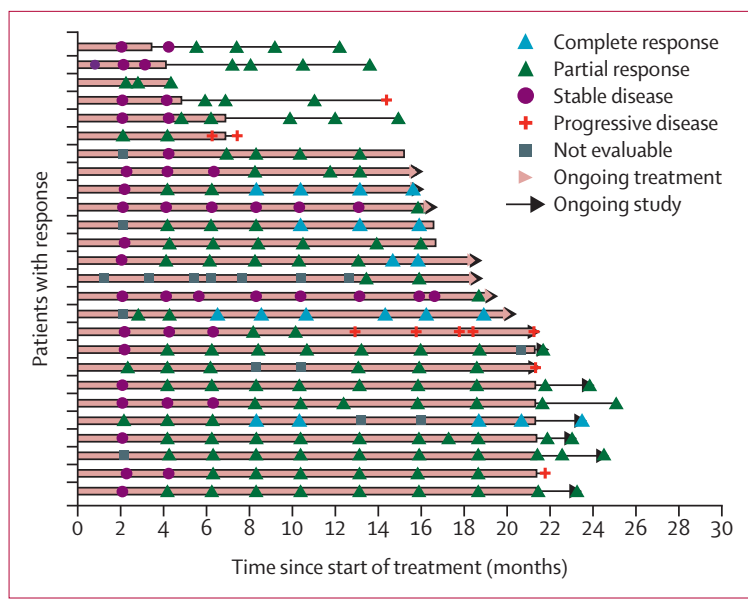

Figure 1: Tumour response to cemiplimab per independent central review Response and duration of response in patients with confirmed complete response or partial response by independent central review (26 patients with confirmed responses, including two partial responses that emerged at tumour assessments before data cutoff but were confirmed by tumour assessments performed subsequent to data cutoff). 15 patients were still on the study at the time of data cutoff.

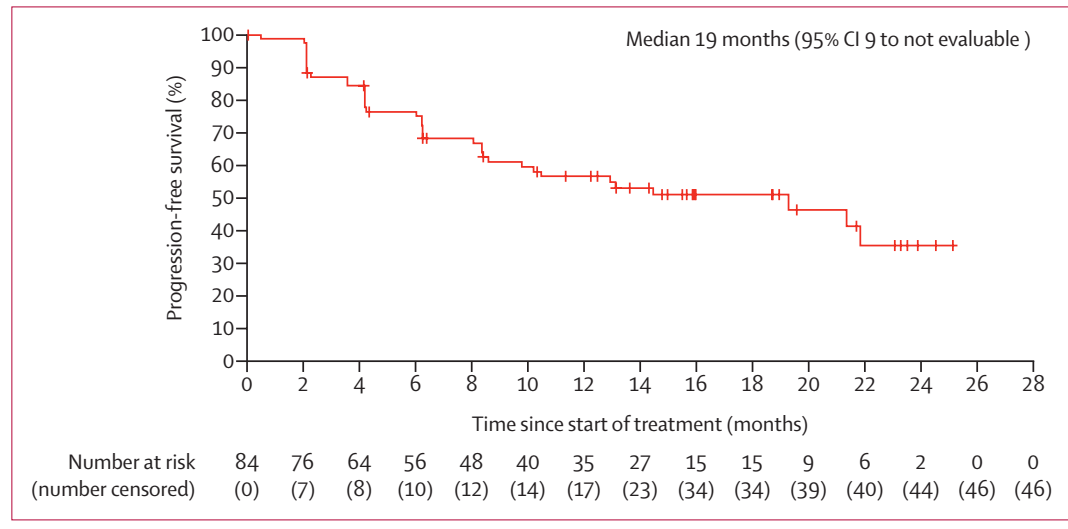

Figure 2: Kaplan-Meier curve for progression-free survival per independent central review Crosses denote censored patients.
Tumour responses per independent central review are shown in figure 1 . Among the 26 patients with confirmed responses by ICR, only five had evidence of subsequent disease progression. Changes in target lesions over time is shown in the appendix (p 13).

By ICR, with 38 events (including 33 patients with progressive disease and five deaths), the median KaplanMeier estimation of progression-free survival was 19 months (95\% CI 9-not evaluable). The Kaplan-Meier estimated proportion of patients alive and without disease progression at 6 months was $76 \%$ (95\% CI 65-84) and at 12 months was 57\% (44-67; figure 2). Progression-free survival by investigator assessment is shown in the appendix ( $p$ 14). Median overall survival had not been reached at the time of data cutoff. With ten events, Kaplan-Meier estimated proportion of patients alive at 2 years was 80\% (95\% CI 63-90; appendix p 15).

In subgroup analyses, clinical activity was similar regardless of baseline characteristics (appendix p 16).

Baseline tumour samples were evaluable for PD-L1 immunohistochemistry in $50(60 \%)$ of 84 patients, TMB assessments in $56(66 \%)$ of 84 patients, and MHC-I immunohistochemistry in $44(52 \%)$ of 84 patients. Exploratory biomarker data showed no clinically meaningful associations between objective response and any of the following candidate biomarkers: PD-L1 expression (appendix p 9), TMB (appendix p 17), or MHC-I expression (appendix p 18). Among some patients with high TMB who did not have objective responses, MHC-I expression level on tumour cells was low or absent (appendix p 19).

Grade 3-4 treatment-emergent adverse events occurred in $40(48 \%)$ of 84 patients with the most common being hypertension and colitis each in four (5\%) patients, and fatigue, urinary tract infection, and visual impairment each occurring in three patients (4\%; table 3$)$. One $(1 \%)$ of 84 patients had a treatment dose reduction due to a grade 3 cutaneous soft tissue infection of basal cell carcinoma that was considered unrelated to study treatment. Nine (11\%) of 84 patients discontinued treatment due to the following treatment-related adverse events, each in one patient: grade 3 adrenal insufficiency, grade 3 asthenia, grade 3 colitis, grade 3 hypophysitis, grade 3 immune-mediated hepatitis, grade 2 acute kidney injury, grade 2 colitis, grade 2 enterocolitis, grade 2 immune-related hypothyroidism, grade 1 colitis, and grade 1 renal failure (some patients had more than one treatment-related adverse events that led to discontinuation). Serious treatmentemergent adverse events occurred in 29 (35\%) of 84 patients, with nine $(11 \%)$ considered related to study treatment; the most common serious treatment-related adverse events were colitis in three $(4 \%)$ patients and adrenal insufficiency in two (2\%). Treatment-related adverse events are summarised in the appendix (p 10).

Sponsor-identified immune-related adverse events occurred in 21 (25\%) of 84 patients (appendix p 11). The most common were hypothyroidism in eight $(10 \%)$ patients 


\begin{tabular}{|lclll|}
\hline & Grade 1-2 & Grade 3 & Grade 4 & Grade 5 \\
\hline Any & $39(46 \%)$ & $38(45 \%)$ & $2(2 \%)$ & $3(4 \%)$ \\
\hline Fatigue & $22(26 \%)$ & $3(4 \%)$ & 0 & 0 \\
\hline Diarrhoea & $20(24 \%)$ & 0 & 0 & 0 \\
\hline Pruritus & $18(21 \%)$ & 0 & 0 & 0 \\
\hline Asthenia & $16(19 \%)$ & $1(1 \%)$ & 0 & 0 \\
\hline Anaemia & $12(14 \%)$ & $1(1 \%)$ & 0 & 0 \\
Decreased appetite & $12(14 \%)$ & $1(1 \%)$ & 0 & 0 \\
\hline Arthralgia & $11(13 \%)$ & 0 & 0 & 0 \\
Headache & $11(13 \%)$ & $1(1 \%)$ & 0 & 0 \\
\hline Nausea & $11(13 \%)$ & $1(1 \%)$ & 0 & 0 \\
\hline Dyspnoea & $10(12 \%)$ & 0 & 0 & 0 \\
\hline Urinary tract infection & $9(11 \%)$ & $3(4 \%)$ & 0 & 0 \\
Cough & $8(10 \%)$ & 0 & 0 & 0 \\
\hline Dizziness & $8(10 \%)$ & 0 & 0 & 0 \\
Hypothyroidism & $8(10 \%)$ & 0 & 0 & 0 \\
\hline Increased blood creatinine & $8(10 \%)$ & 0 & 0 & 0 \\
\hline Tumour haemorrhage & $8(10 \%)$ & 0 & 0 & 0 \\
\hline Leukocytosis & $6(7 \%)$ & $1(1 \%)$ & 0 & 0 \\
\hline Basal cell carcinoma & $5(6 \%)$ & $1(1 \%)$ & 0 & 0 \\
\hline Bronchitis & $5(6 \%)$ & $1(1 \%)$ & 0 & 0 \\
\hline Decreased weight & $5(6 \%)$ & $2(2 \%)$ & 0 & 0 \\
\hline Abdominal pain & $4(5 \%)$ & $2(2 \%)$ & 0 & 0 \\
\hline Constipation & $4(5 \%)$ & $1(1 \%)$ & 0 & 0 \\
\hline Hypalbuminaemia & $4(5 \%)$ & $1(1 \%)$ & 0 & 0 \\
\hline Maculopapular rash & $4(5 \%)$ & $1(1 \%)$ & 0 & 0 \\
\hline Hypokalaemia & $3(4 \%)$ & $1(1 \%)$ & 0 & 0 \\
\hline Dysphagia & $3(4 \%)$ & $1(1 \%)$ & 0 & 0 \\
\hline Hypertension & $3(4 \%)$ & $4(5 \%)$ & 0 & 0 \\
\hline Lymphopenia & $3(4 \%)$ & $1(1 \%)$ & 0 & 0 \\
\hline Somnolence & $3(4 \%)$ & $1(1 \%)$ & 0 & 0 \\
\hline Hyperkalaemia & $2(2 \%)$ & $1(1 \%)$ & 0 & 0 \\
\hline Hyponatraemia & $2(2 \%)$ & $2(2 \%)$ & 0 & 0 \\
\hline Infected neoplasm & $2(2 \%)$ & $2(2 \%)$ & 0 & 0 \\
\hline Skin ulcer & $2(2 \%)$ & $1(1 \%)$ & 0 & 0 \\
\hline Acute kidney injury & $1(1 \%)$ & 0 & 0 & $1(1 \%)$ \\
\hline Adrenal insufficiency & $1(1 \%)$ & $2(2 \%)$ & 0 & 0 \\
Cholestasis & $1(1 \%)$ & $1(1 \%)$ & 0 & 0 \\
\hline Ear infection & $1(1 \%)$ & $1(1 \%)$ & 0 & 0 \\
Eye pain & $1(1 \%)$ & $1(1 \%)$ & 0 & 0 \\
\hline General physical health & $1(1 \%)$ & $1(1 \%)$ & 0 & 0 \\
deterioration & & & & \\
Hypotension & $1(1 \%)$ & $1(1 \%)$ & 0 & 0 \\
\hline Increased blood pressure & $1(1 \%)$ & $2(2 \%)$ & 0 & 0 \\
\hline Increased gamma- & $1(1 \%)$ & $1(1 \%)$ & 0 & 0 \\
glutamyl transferase & & & & \\
& & & & \\
\hline
\end{tabular}

and immune-related colitis in three (4\%) patients. Immune-related adverse events were grade 3 in eight (10\%) patients. Grade 3 immune-related adverse events that occurred in more than one patient were immunerelated colitis in three patients and adrenal insufficiency in two patients. There were no grade 4 or grade 5 immune-related adverse events.

\begin{tabular}{|c|c|c|c|c|}
\hline & Grade 1-2 & Grade 3 & Grade 4 & Grade 5 \\
\hline \multicolumn{5}{|c|}{ (Continued from previous column) } \\
\hline Increased weight & $1(1 \%)$ & $1(1 \%)$ & 0 & 0 \\
\hline Pneumonitis & $1(1 \%)$ & $1(1 \%)$ & 0 & 0 \\
\hline Tumour pain & $1(1 \%)$ & $1(1 \%)$ & 0 & 0 \\
\hline $\begin{array}{l}\text { Abnormal general } \\
\text { physical condition }\end{array}$ & 0 & $1(1 \%)$ & 0 & 0 \\
\hline Brain oedema & 0 & $1(1 \%)$ & 0 & 0 \\
\hline Cachexia & 0 & 0 & 0 & $1(1 \%)$ \\
\hline Calculus bladder & 0 & $1(1 \%)$ & 0 & 0 \\
\hline $\begin{array}{l}\text { Cerebrospinal fluid } \\
\text { leakage }\end{array}$ & 0 & $1(1 \%)$ & 0 & 0 \\
\hline Cerebrovascular accident & 0 & $1(1 \%)$ & 0 & 0 \\
\hline Colitis* & 0 & $4(5 \%)$ & 0 & 0 \\
\hline Corneal abscess & 0 & $1(1 \%)$ & 0 & 0 \\
\hline $\begin{array}{l}\text { Decreased injection } \\
\text { fraction }\end{array}$ & 0 & $1(1 \%)$ & 0 & 0 \\
\hline Decreased mood & 0 & $1(1 \%)$ & 0 & 0 \\
\hline Delirium & 0 & $1(1 \%)$ & 0 & 0 \\
\hline Ear disorder & 0 & $1(1 \%)$ & 0 & 0 \\
\hline Erosive gastritis & 0 & $1(1 \%)$ & 0 & 0 \\
\hline Hypophysitis & 0 & $1(1 \%)$ & 0 & 0 \\
\hline $\begin{array}{l}\text { Immune-mediated } \\
\text { hepatitis }\end{array}$ & 0 & $1(1 \%)$ & 0 & 0 \\
\hline $\begin{array}{l}\text { Lower respiratory tract } \\
\text { infection }\end{array}$ & 0 & $1(1 \%)$ & 0 & 0 \\
\hline $\begin{array}{l}\text { Malignant brain } \\
\text { neoplasm }\end{array}$ & 0 & 0 & 0 & $1(1 \%)$ \\
\hline Myocardial infarction & 0 & 0 & $1(1 \%)$ & 0 \\
\hline Phlebitis & 0 & $1(1 \%)$ & 0 & 0 \\
\hline Pleural embolism & 0 & $1(1 \%)$ & 0 & 0 \\
\hline Retinal vein thrombosis & 0 & $1(1 \%)$ & 0 & 0 \\
\hline Soft tissue infection & 0 & $1(1 \%)$ & 0 & 0 \\
\hline $\begin{array}{l}\text { Supraventricular } \\
\text { tachycardia }\end{array}$ & 0 & $2(2 \%)$ & 0 & 0 \\
\hline $\begin{array}{l}\text { Transient ischaemic } \\
\text { attack }\end{array}$ & 0 & $1(1 \%)$ & 0 & 0 \\
\hline Upper limb fracture & 0 & $1(1 \%)$ & 0 & 0 \\
\hline Visual impairment & 0 & $2(2 \%)$ & $1(1 \%)$ & 0 \\
\hline \multicolumn{5}{|c|}{$\begin{array}{l}\text { Data are } \mathrm{n}(\%) \text { in all treated patients. Treatment-emergent adverse events, } \\
\text { regardless of attribution, reported in at least } 10 \% \text { of patients (grades } 1-2 \text { ) or by } \\
\text { any patient (grades 3-5) are shown. *Includes preferred terms colitis and } \\
\text { autoimmune colitis. }\end{array}$} \\
\hline
\end{tabular}

There were no treatment-related deaths. Three deaths were reported that were due to treatment-emergent adverse events, but they were considered as related to intercurrent medical issues. They included one patient with new intracranial sarcoma arising from transformation of known meningioma; one patient with acuteon-chronic renal failure in the setting of suspected septic pneumonia; and one patient with history of malnutrition, who died due to cachexia that was reported as worsening constitutional syndrome. The patient who died due to cachexia also experienced 
grade 3 immune-related colitis that resolved before his death.

\section{Discussion}

The landmark studies of immune checkpoint blockade in melanoma were followed by demonstrations that PD-1 or PD-L1 blockade is a highly active therapy against advanced CSCC and Merkel cell carcinoma. ${ }^{14-16,21}$ Until Feb 9, 2021, when cemiplimab was approved by the US Food and Drug Administration fully for patients with locally advanced basal cell carcinoma, and accelerated for patients with metastatic basal cell carcinoma, after HHI treatment, or for whom HHIs are not appropriate, there was no standard treatment regimen after first-line $\mathrm{HHI}$ therapy for patients with locally advanced basal cell carcinoma. To our knowledge, this is the first multicentre, prospective study of checkpoint blockade in patients with basal cell carcinoma, and the first pivotal study of a treatment option for patients with basal cell carcinoma after HHIs. This study shows clinically meaningful antitumour activity of cemiplimab in patients with locally advanced basal cell carcinoma after HHI therapy. Centrally reviewed objective response was observed in 31\% (95\% CI 21-42) of patients with estimated duration of response exceeding 1 year in $85 \%$ of responders. The safety profile was consistent with what is known for the anti-PD-1 or anti-PD-L1 class of drugs, even considering the advanced age of the patient population in the present study.

These results fill a long-standing gap regarding the absence of treatment options for patients with locally advanced basal cell carcinoma after first-line $\mathrm{HHI}$ therapy. Objective responses with HHI therapy occur in approximately half of patients with locally advanced basal cell carcinoma, but most do not have complete responses.,22 Among those patients who respond to HHI therapy, the median duration of response was 26 months, underscoring the development of resistance to HHI therapy leading to loss of response. ${ }^{6,7}$ Toxicities of the HHI class included dysgeusia, muscle spasms, and alopecia. Although toxicity was the most common reason for treatment discontinuation in the largest prospective studies of vismodegib, ${ }^{23,24}$ the most common reason for discontinuation of previous HHI therapy in the current study was disease progression. Therefore, the patient population enrolled in this cemiplimab study represents an unequivocal unmet need. In the pivotal clinical studies leading to approval of HHIs for the treatment of locally advanced basal cell carcinoma, survival outcomes were not reported for the subset of patients with disease progression on HHIs. ${ }^{47}$ To our knowledge, this study is the first demonstration of clinical benefit for any systemic therapy for patients with locally advanced basal cell carcinoma after HHI. Clinically meaningful activity of cemiplimab in both basal cell carcinoma and CSCC was consistent with the shared clinical and molecular characteristics of these keratinocyte carcinomas. ${ }^{25}$
The kinetics of response to cemiplimab were slower in patients with basal cell carcinoma than those with CSCC, suggesting that a longer duration of treatment could be required for some patients with basal cell carcinoma to have an optimal tumour response. Median time to response was 2 months in patients with advanced CSCC treated with cemiplimab, ${ }^{15,16}$ but was 4.3 months (IQR 4.2-7.2) in the present study in patients with locally advanced basal cell carcinoma. Responses to cemiplimab in both tumour types showed durability, which was conclusively established in the long-term follow-up in the CSCC study. ${ }^{26}$ Some partial responses mature into complete responses in patients with CSCC. ${ }^{16}$ At the most recent update of the pivotal CSCC study, the group with the longest follow-up (group 1, median follow-up 19 months) showed complete response in 20\% of the patients, compared with $7 \%$ at the time of primary analysis when median follow-up was 8 months. ${ }^{26}$ Active follow-up of patients with locally advanced basal cell carcinoma continues in this study, and some of the current partial responses could evolve into complete responses with continued follow-up.

Exploratory correlative biomarker analyses in this study did not support the use of PD-L1 or TMB to predict response to or clinical benefit of cemiplimab in locally advanced basal cell carcinoma. A recent study ${ }^{27}$ of the antiPD-1 drug, pembrolizumab, in multiple solid tumour types defined high TMB as at least 10 mut/MB. However, the present study showed that some high TMB tumours (at least $10 \mathrm{mut} / \mathrm{MB}$ ) do not respond to treatment and some low TMB tumours do respond to cemiplimab. The observation of low or absent MHC-I expression among a subgroup of non-responders provides, to our knowledge, the first prospective evidence that MHC-I downregulation, previously described retrospectively in selected basal cell carcinoma tumours, ${ }^{28}$ is a potential mechanism of immune evasion during anti-PD-1 therapy of high TMB tumours. Mechanisms of MHC-I downregulation in basal cell carcinoma require further investigation, because the optimal therapeutic approach in future clinical trials depends on whether reduced MHC-I expression in basal cell carcinoma is reversible for therapeutic advantage and synergistic with immunotherapy. Additional studies are needed to determine if these observations regarding MHC-I downregulation in basal cell carcinoma also occur in other tumour types with high TMB

There is an emerging paradigm that clinical activity of immunotherapy is greatest when administered early in the natural history of cancers. ${ }^{29}$ Future clinical study should evaluate cemiplimab before HHI therapy. Concurrent HHI plus cemiplimab might not be optimal, because preclinical data show that blockade of smoothened signalling inhibits formation of the immunological synapse..$^{30} \mathrm{~A}$ pilot study of vismodegib in combination with pembrolizumab did not suggest additive clinical activity. ${ }^{31}$

One of the limitations of the present report from this study is the lack of longer-term follow-up data. These 
data are being collected and will further characterise the clinical activity and duration of response to cemiplimab in patients with locally advanced basal cell carcinoma. Other limitations include the small number of patients in this study and the single-arm design. Regardless of these limitations, cemiplimab showed clinical activity in a patient group who, until recently, had no standard treatment option after HHI therapy.

In conclusion, to our knowledge, this cemiplimab study is the first to show clinical benefit of a systemic therapy, and supports the recent indication of cemiplimab in the USA as the first immunotherapy, for patients with locally advanced basal cell carcinoma after HHI therapy or for whom HHIs are not appropriate.

\section{Contributors}

AJS, AS, KP, KDL, FS, GDY, IL, and MGF conceived and designed the study. TB worked on the study design and amendments. AJS, KP, OB, SPr, MK, KDL, NB-S, ALSC, SD, AFO, LL, CR, CU, AH, MRM, RD, IDB, CL, VDG, ZE, RG, JU, and SPu recruited patients and collected the data. SL and S-YY analysed the data. KM, EC, and DMW contributed to the overall data analysis. VJ, NF, and GT worked on biomarker study analysis. EO worked on safety analysis strategy and contributed to safety data analysis. All authors had full access to and verified the data, contributed to the data interpretation, as well as critical review, revision, and approval of, and the decision to submit the report for publication.

All authors had unrestricted access to study data, were responsible for all content and editorial decisions, and received no honoraria related to the development of this publication.

\section{Declaration of interests}

AJS reports advisory board or steering committee roles with Janssen, Regeneron Pharmaceuticals, Roche, and Sanofi; and research support from Abbvie, Bristol Myers Squibb, Genesis Pharma, and Novartis. AS reports advisory roles with Regeneron Pharmaceuticals and Roche. KP reports advisory board roles with Abbvie, LEO Pharma, Janssen, Almirall, Eli Lilly, Galderma, Novartis, Pierre Fabre, Sun Pharma, and Sanofi, outside the submitted work. OB has advisory board roles with Novartis, Bristol Myers Squibb, Merck Sharp \& Dohme, Pierre Fabre, and Ultimovacs. KDL reports grants from the University of Colorado; and grants and personal fees from Regeneron Pharmaceuticals. ALSC reports advisory roles with Regeneron Pharmaceuticals and Merck; and research funding from Regeneron Pharmaceuticals, Merck, Novartis, and Galderma. SD reports that their spouse is an employee of Sanofi. LL reports institutional grants and personal fees from AstraZeneca, Bristol Myers Squibb, Boehringer Ingelheim, Debiopharm International SA, Eisai, Novartis, and Roche; institutional grants from Celgene International, Exelixis, Hoffmann-La Roche, IRX Therapeutics, Medpace, Merck-Serono, and Pfizer; and personal fees from Sobi, Ipsen, Incyte Biosciences Italy SRL, Doxa Pharma, Amgen, Nanobiotics SA, GlaxoSmithKline, AccMed, Medical Science Fundation G. Lorenzini, Associazione Sinapsi, Think 2 IT, Aiom Servizi, Prime Oncology, WMA Congress Education, Fasi, DueCi Promotion SRL, MI\&T, Net Congress \& Education, PRMA Consulting, Kura Oncology, Health \& Life SRL, and Immuno-Oncology Hub. CR reports grants and personal fees advisory board roles with Bristol Myers Squibb, Pierre Fabre, Novartis, Amgen, Merck, Roche, Merck Sharp \& Dohme, Sanofi, Biothera, and Ultimovacs. CU reports advisory board and speaker roles for Roche, Sanofi, Regeneron Pharmaceuticals, and Sun Pharma. AH reports institutional funding and personal fees from Amgen, Bristol Myers Squibb, Merck Serono, Merck Sharp \& Dohme, Merck \& Co, Philogen, Pierre Fabre, Provectus, Regeneron Pharmaceuticals, Roche, Sanofi Genzyme, and Novartis; and consultancy fees from OncoSec, Almirall Hermal, and Sun Pharma. MRM reports advisory roles with and travel fees from Regeneron Pharmaceuticals and Sun Pharmaceuticals; advisory role with Rakuten Medical; and research funding from Regeneron Pharmaceuticals and PellePharm. RD has intermittent, project focused consultant and advisory roles with Novartis, Merck Sharp \& Dohme, Bristol Myers Squibb, Roche, Amgen, Takeda,
Pierre Fabre, Sun Pharma, Sanofi, Catalym, Second Genome, Regeneron Pharmaceuticals, Alligator, MaxiVAX SA, and touchIME outside the submitted work. SL, S-YY, KM, EC, VJ, NF, EO, FS, DMW, and TB are employees and shareholders of Regeneron Pharmaceuticals. CL reports personal fees from Roche, Sun Pharma, Bristol Myers Squibb, Merck Sharp \& Dohme, Merck, Novartis, Pierre Fabre, Kyowa Kirin, Almiral Hermal, and Biontech. ZE reports advisory board fees from Regeneron Pharmaceuticals, Novartis, Array, Genentech, and SunPharma; and grants from Novartis. RG reports documentation fees to institution from Regeneron Pharmaceuticals; personal fees and non-financial support from Amgen, Bristol Myers Squibb, Roche Pharma, Merck Serono, Pierre Fabre, Sanofi, and Regeneron Pharmaceuticals; grants, personal fees, and non-financial support from Novartis; grants and personal fees from Pfizer; grants from Johnson \& Johnson; and personal fees from Merck Sharp \& Dohme, Almirall Hermal, SUN Pharma, 4SC, and Bayer. JU reports grants and personal fees from Sanofi, Bristol Myers Squibb, Novartis, and Merck Sharp \& Dohme; and personal fees from Roche, medac, and Sun Pharma. SPu reports personal fees and non-financial support from Sanofi; other (clinical trials) from Regeneron Pharmaceuticals; grants from Avene; non-financial support from Abbie; grants, personal fees, and non-financial support from ISDIN, La Roche-Posay, and Roche; grants and personal fees from Sun Pharma; non-financial support from Eli Lilly and Merck Sharp \& Dohme; personal fees, non-financial support, and other (related to clinical trials) from Pfizer; grants, personal fees, and other from Leo Pharma and Almirall; personal fees and other (related to clinical trials) from Ojer Pharma; personal fees from Pierre Fabre; and non-financial support from MAVIG, FotoFinder, and $3 \mathrm{Gen}$. GDY is an employee of, shareholder in, and a member of the Board of Directors at Regeneron Pharmaceuticals. GT is an employee of, patent holder, and shareholder of Regeneron Pharmaceuticals. IL and MGF are employees of, have patents pending, and are shareholders of Regeneron Pharmaceuticals. SPr, MK, NB-S, AFO, IB, and VDG declare no competing interests.

\section{Data sharing}

Qualified researchers may request access to study documents (including the clinical study report, study protocol with any amendments, blank case report form, and statistical analysis plan) that support the methods and findings reported in this manuscript. Individual anonymised participant data will be considered for sharing once the product and indication has been approved by major health authorities (eg, Food and Drug Administration, European Medicines Agency, Pharmaceuticals, and Medical Devices Agency, etc), if there is legal authority to share the data, and there is not a reasonable likelihood of participant re-identification. Requested should be submitted to https://vivli.org/.

\section{Acknowledgments}

This study was funded by Regeneron Pharmaceuticals and Sanofi. The authors would like to thank the patients, their families, all other investigators, and all investigational site members involved in this study. The authors would also like to thank Sara Hamon from Regeneron Pharmaceuticals for assistance with statistical analysis. Medical writing support under the direction of the authors was provided by Emmanuel Ogunnowo of Prime (Knutsford, UK) and funded by Regeneron Pharmaceuticals according to Good Publication Practice guidelines. The sponsor was involved in the study design, collection, analysis, and interpretation of data, as well as data checking of information provided in the manuscript.

\section{References}

1 Puig S, Berrocal A. Management of high-risk and advanced basal cell carcinoma. Clin Transl Oncol 2015; 17: 497-503.

2 Wu S, Han J, Li W-Q, Li T, Qureshi AA. Basal-cell carcinoma incidence and associated risk factors in U.S. women and men. Am J Epidemiol 2013; 178: 890-97.

3 Migden MR, Chang ALS, Dirix L, Stratigos AJ, Lear JT. Emerging trends in the treatment of advanced basal cell carcinoma. Cancer Treat Rev 2018; 64: 1-10.

4 Sekulic A, Migden MR, Oro AE, et al. Efficacy and safety of vismodegib in advanced basal-cell carcinoma. N Engl J Med 2012; 366: 2171-79. 
5 Migden MR, Guminski A, Gutzmer R, et al. Treatment with two different doses of sonidegib in patients with locally advanced or metastatic basal cell carcinoma (BOLT): a multicentre, randomised, double-blind phase 2 trial. Lancet Oncol 2015; 16: 716-28.

6 Sekulic A, Migden MR, Basset-Seguin N, et al. Long-term safety and efficacy of vismodegib in patients with advanced basal cell carcinoma: final update of the pivotal ERIVANCE BCC study. BMC Cancer 2017; 17: 332 .

7 Dummer R, Guminksi A, Gutzmer R, et al. Long-term efficacy and safety of sonidegib in patients with advanced basal cell carcinoma: 42-month analysis of the phase II randomized, double-blind BOLT study. Br J Dermatol 2020; 182: 1369-78.

8 Chang ALS, Oro AE. Initial assessment of tumor regrowth after vismodegib in advanced Basal cell carcinoma. Arch Dermatol 2012; 148: 1324-25.

9 Chalmers ZR, Connelly CF, Fabrizio D, et al. Analysis of 100,000 human cancer genomes reveals the landscape of tumor mutational burden. Genome Med 2017; 9: 34.

10 Büttner R, Longshore JW, López-Ríos F, et al. Implementing TMB measurement in clinical practice: considerations on assay requirements. ESMO Open 2019; 4: e000442.

11 Euvrard S, Kanitakis J, Claudy A. Skin cancers after organ transplantation. N Engl J Med 2003; 348: 1681-91.

12 Murphy AJ, Macdonald LE, Stevens S, et al. Mice with megabase humanization of their immunoglobulin genes generate antibodies as efficiently as normal mice. Proc Natl Acad Sci USA 2014; 111: 5153-58.

13 Macdonald LE, Karow M, Stevens S, et al. Precise and in situ genetic humanization of $6 \mathrm{Mb}$ of mouse immunoglobulin genes. Proc Natl Acad Sci USA 2014; 111: 5147-52.

14 Migden MR, Rischin D, Schmults CD, et al. PD-1 blockade with cemiplimab in advanced cutaneous squamous-cell carcinoma. N Engl J Med 2018; 379: 341-51.

15 Migden MR, Khushalani NI, Chang ALS, et al. Cemiplimab in locally advanced cutaneous squamous cell carcinoma: results from an openlabel, phase 2, single-arm trial. Lancet Oncol 2020; 21: 294-305.

16 Rischin D, Migden MR, Lim AM, et al. Phase 2 study of cemiplimab in patients with metastatic cutaneous squamous cell carcinoma: primary analysis of fixed-dosing, long-term outcome of weight-based dosing. J Immunother Cancer 2020; 8: e000775.

17 Sezer A, Kilickap S, Gümüş M, et al. Cemiplimab monotherapy for first-line treatment of advanced non-small-cell lung cancer with PD-L1 of at least 50\%: a multicentre, open-label, global, phase 3 randomised, controlled trial. Lancet 2021; 397: 592-604.

18 Falchook GS, Leidner R, Stankevich E, et al. Responses of metastatic basal cell and cutaneous squamous cell carcinomas to anti-PD1 monoclonal antibody REGN2810. J Immunother Cancer 2016; 4: 70.
19 Eisenhauer EA, Therasse P, Bogaerts J, et al. New response evaluation criteria in solid tumours: revised RECIST guideline (version 1.1). Eur J Cancer 2009; 45: 228-47.

20 Clopper CJ, Pearson ES. The use of confidence or fiducial limits illustrated in the case of the binomial. Biometrika 1934; 26: 404-13.

21 Barrios DM, Do MH, Phillips GS, et al. Immune checkpoint inhibitors to treat cutaneous malignancies. J Am Acad Dermatol 2020; 83: 1239-53.

22 Sekulic A, Migden MR, Lewis K, et al. Pivotal ERIVANCE basal cell carcinoma (BCC) study: 12-month update of efficacy and safety of vismodegib in advanced BCC. J Am Acad Dermatol 2015; 72: 1021-26.

23 Basset-Séguin N, Hauschild A, Kunstfeld R, et al. Vismodegib in patients with advanced basal cell carcinoma: Primary analysis of STEVIE, an international, open-label trial. Eur J Cancer 2017; 86: $334-48$.

24 Dréno B, Kunstfeld R, Hauschild A, et al. Two intermittent vismodegib dosing regimens in patients with multiple basal-cell carcinomas (MIKIE): a randomised, regimen-controlled, double-blind, phase 2 trial. Lancet Oncol 2017; 18: 404-12.

25 Nehal KS, Bichakjian CK. Update on keratinocyte carcinomas. N Engl J Med 2018; 379: 363-74.

26 Rischin D, Khushalani NI, Schmults CD, et al. Phase 2 study of cemiplimab in patients with advanced cutaneous squamous cel carcinoma (CSCC): longer follow-up. 10th World Congress of Melanoma in conjuction with 17th EADO Congress; Rome, Italy; April 15-17, 2021 (abstr p-236).

27 Marabelle A, Fakih M, Lopez J, et al. Association of tumour mutational burden with outcomes in patients with advanced solid tumours treated with pembrolizumab: prospective biomarker analysis of the multicohort, open-label, phase 2 KEYNOTE-158 study. Lancet Oncol 2020; 21: 1353-65.

28 Walter A, Barysch MJ, Behnke S, et al. Cancer-testis antigens and immunosurveillance in human cutaneous squamous cell and basal cell carcinomas. Clin Cancer Res 2010; 16: 3562-70.

29 Topalian SL, Taube JM, Pardoll DM. Neoadjuvant checkpoint blockade for cancer immunotherapy. Science 2020; 367: eaax0182.

30 de la Roche M, Ritter AT, Angus KL, et al. Hedgehog signaling controls T cell killing at the immunological synapse. Science 2013 342: $1247-50$.

31 Chang ALS, Tran DC, Cannon JGD, et al. Pembrolizumab for advanced basal cell carcinoma: An investigator-initiated, proof-ofconcept study. J Am Acad Dermatol 2019; 80: 564-66. 